\title{
Repensar los cuidados a la luz de la reproducción social: una propuesta para vincular los circuitos migratorios $^{1}$
}

\author{
Eleonore KOFMAN \\ Middlesex University of London \\ e.kofman@mdx.ac.uk
}

Recibido: Enero 2016

Aceptado (traducción): Enero 2016

\section{RESUMEN}

Los cuidados se han convertido en un elemento predominante en gran parte de las investigaciones feministas sobre migraciones globalizadas y la transferencia de trabajo del Sur al Norte. El concepto de reproducción, en cambio, ha sido relegado durante años a un segundo plano, aunque ahora se está convirtiendo en el foco de los debates sobre la mercantilización de los cuidados en el hogar y las transformaciones de las políticas del estado de bienestar. Este artículo sostiene que, si vinculamos los diversos circuitos de migración y, sobre todo, los relacionados con el empleo y la familia, podremos comprender mejor las diferentes formas y recorridos de los cuidados en la reproducción de personas, familias y comunidades, tanto migrantes como no migrantes. Para ello, me retrotraigo a los trabajos estadounidenses sobre minorías racializadas y migrantes, así como sobre reproducción social estratificada. Asimismo, tomo algunas ideas de los actuales estudios asiáticos sobre circuitos migratorios generizados que establecen vínculos entre las migraciones por empleo y por matrimonio. También uso la noción de ‘familias transnacionales' que pone de manifiesto cómo operan las políticas de género de la reproducción social, tanto en el seno como más allá de las familias, los hogares y las estructuras institucionales y de bienestar. Al contrario que en Asia, los estudios europeos apenas se han centrado en la vinculación que existe entre las migraciones laborales y las familiares desde el punto de vista de la reproducción social. Sin embargo, relacionar los diferentes tipos de migraciones permite comprender en su complejidad las trayectorias de los cuidados y el modo en que éstas contribuyen a la reproducción social.

Palabras clave: Migraciones, género, (trayectorias de) cuidados, reproducción (social), familias transnacionales.

\footnotetext{
${ }^{1}$ Translated by Itzea Goicolea-Amiano. Original version in English published in Social Politics 2012, vol. 19, n. 1, pp. 142-162. doi:10.1093/sp/jxr030
} 


\section{Rethinking Care Through Social Reproduction: Articulating Circuits of Migration}

\section{ABSTRACT}

Care has come to dominate much feminist research on globalized migrations and the transfer of labor from the South to the North, while the older concept of reproduction had been pushed into the background but is now becoming the subject of debates on the commodification of care in the household and changes in welfare state policies. This article argues that we could achieve a better understanding of the different modalities and trajectories of care in the reproduction of individuals, families, and communities, both of migrant and nonmigrant populations by articulating the diverse circuits of migration, in particular that of labor and the family. In doing this, I go back to the earlier North American writing on racialized minorities and migrants and stratified social reproduction. I also explore insights from current Asian studies of gendered circuits of migration connecting labor and marriage migrations as well as the notion of global householding that highlights the gender politics of social reproduction operating within and beyond households in institutional and welfare architectures. In contrast to Asia, there has relatively been little exploration in European studies of the articulation of labor and family migrations through the lens of social reproduction. However, connecting the different types of migration enables us to achieve a more complex understanding of care trajectories and their contribution to social reproduction.

Keywords: Migration, gender, care (trajectories), (social) reproduction, global householding.

\section{INTRODUCCIÓN}

Conforme el estudio de los cuidados ha ido prevaleciendo en las investigaciones feministas en torno a las migraciones globalizadas y la transferencia del trabajo del Sur al Norte, el uso del concepto de reproducción social, anterior al de los cuidados, ha ido disminuyendo y siendo relegado a un segundo plano. Sin embargo, aunque no se les presta la misma atención, ambos conceptos suelen aparecer relacionados. Concretamente, suele considerarse que el trabajo de cuidado contribuye a la globalización de la reproducción social y, por lo tanto, que es una forma de trabajo reproductivo (Hill Maher, 2004; Misra, Woodring y Merz, 2006; Perez Orozco, 2010). El Instituto de Investigación de las Naciones Unidas para el Desarrollo Social (UNRISD, por sus siglas en inglés; 2009, 8) indica que la economía social y política del cuidado conlleva "conciliar la carga de la reproducción con la de otras tareas sociales, para permitir que se compartan las responsabilidades de la reproducción" entre las y los miembros de la sociedad.

Los cuidados bien podrían definirse como una variedad de actividades y relaciones que promueven el bienestar físico y emocional de personas "que no pueden o no son proclives a realizar dichas actividades por sí mismas” (Yeates, 2004, 371). La noción de reproducción, por otra parte, fue objeto de un extenso debate en la década de los setenta, cuando se hacía hincapié en la contribución del empleo doméstico no remunerado a la reproducción de la fuerza de trabajo (Beneria, 1979; Molyneux, 1979; Vogel, 1995). No obstante, las críticas más feroces (Molyneux, 1979) apuntaron al reduccionismo económico y la abstracción con respecto a especificidades sociales e históricas en que se incurría, así como a la falta de consideración del trabajo dentro del 
hogar -el cual, además, carecía de una contextualización socio-económica amplia y adecuada $^{2}$.

Ello no implica, claro está, que el concepto de reproducción haya quedado en desuso. La economía feminista se ha basado en gran medida en el trabajo no remunerado y en el ámbito doméstico (Engly y Folbre, 2003; Elson, 2000). De hecho, los y las feministas, en especial aquéllas cuya perspectiva se enmarca en la economía política (Bakker, 2007; Bakker y Gill, 2003; Bakker y Silvey, 2008; Bedford y Rai, 2010; Bergeron, 2011; Luxton y Bezanson, 2006), han contribuido a que haya resurgido el interés por la reproducción, que ahora se conceptualiza de manera más amplia y más allá de la mera reproducción de la fuerza de trabajo. Algunas y algunos académicos feministas han usado el estudio de Bourdieu para realizar una aproximación crítica para con los esfuerzos de las mujeres en la reproducción de familias y generaciones a través de diferentes tipos de capital (Adkins y Skeggs, 2005; Reay, 2005). Otros y otras han estudiado la reprivatización de la reproducción que tiene lugar a través de la privatización de propiedades estatales, además de la variedad de formas y grados de la mercantilización del trabajo de cuidado (Bakker, 2007). Por su parte, Bakker y Gill $(2003,18)$ incluyeron en su marco las instituciones encargadas de la socialización del riesgo, la asistencia médica, la educación y otros servicios.

Tanto los procesos biológicos como los de socialización han sido considerados elementos cruciales de la reproducción social ${ }^{3}$. Según Petersen $(2003,79)$, que escribe desde una perspectiva de economía política internacional feminista, la reproducción engloba los procesos materiales y simbólicos necesarios para reproducir seres humanos a lo largo del tiempo (a diario y generacionalmente) en el seno de la familia y el ámbito privado. Peterson analiza la manera en la que el trabajo en el ámbito familiar inscribe códigos, normas de identidad y divisiones de trabajo a través de las prácticas de crianza. Asimismo, cuestiona los supuestos heteronormativos que sustentan la reproducción de las familias ${ }^{4}$. De manera similar, aunque desde un enfoque antropológico, Bjeren $(1997,227)$ diferencia dos procesos: la reproducción de personas en tanto que seres físicos y la reproducción de identidades sociales en el seno de contextos sociales y culturales específicos. Estas dos dimensiones constituyen, pues, la base de la reorganización de las formas familiares y de parentesco, como el matrimonio, tanto en el espacio como en el tiempo. Además, hacen que las relaciones sociales a escala global se establezcan del mismo modo en localidades y estados. Referirse a las familias y al parentesco pone de manifiesto el hecho de que la

\footnotetext{
${ }^{2}$ Resulta especialmente interesante la observación de Molyneux de que el énfasis se ponía en el trabajo que beneficiaba al hombre-sostén de familia más que en la crianza de los y las hijas o en los cuidados. Asimismo, se dejaba a un lado la labor reproductiva ejercida por agentes extrafamiliares.

3 Bledose y Sow (2008) comentan la sorprendente falta de investigación en reproducción (biológica) que se da tanto en antropología como en sociología. Es la disciplina de la demografía la que ha prestado una mayor atención a dicho tema.

${ }^{4}$ La heteronormatividad también puede abordarse de manera crítica en relación a las familias en la bibliografía de las cadenas de cuidados (ver Kofman 2010; Manalansan 2006).
} 
reproducción no sólo tiene lugar a través de procesos laborales y migraciones de corte estrictamente económico, sino también por medio de otros circuitos de migración como el matrimonio que, a su vez, tienen como resultado la creación de nuevas familias y también la reunificación de las que ya existían previamente.

Así pues, a pesar de que el interés en la reproducción social ha resurgido en la economía política feminista y en las disciplinas de la geografía y la sociología, no ha sido así en el campo de la migración generizada, en el que apenas se ha investigado en este sentido. Aunque Bakker y Silvey (2008) y Beneria (2007) entienden la reproducción como un aspecto más de la globalización de la reproducción social, quienes se han dedicado a este tema han solido inclinarse por los cuidados como principal objeto de estudio, dejando las complejidades de la reproducción social como mero telón de fondo.

Mi propuesta, en cambio, es que emplear el concepto de reproducción social nos permite situar los cuidados dentro de un conjunto más amplio de actividades y ámbitos. También nos posibilita establecer vínculos entre los supuestamente dispares circuitos de migración (el empleo, la familia y la educación, en especial), que por lo general se analizan por separado aunque, de hecho, estén relacionados. Y es que todos estos circuitos configuran de diferente manera la reproducción social, la cual puede iniciarse por medio de distintos flujos migratorios. Así, por un lado, la migración por matrimonio puede dar lugar a nuevas familias y conllevar responsabilidades de cuidado para la o el cónyuge de funcionalidad corporal normativa y los y las hijas. A la hora de buscar empleo, es posible que los cónyuges (sobre todo las mujeres) acudan a familiares y que, a su vez, éstas o éstos se desplacen para cuidar de las y los nietos. Por otro lado, la migración por motivos económicos constituye una tipología distinta en la cual la persona migrada cuida de no migrantes además de su familia, tanto en países emisores como en receptores. Así, si no establecemos uniones entre los diferentes circuitos migratorios (por familia y por empleo), nos estamos circunscribiendo a una epistemología generizada y tradicional de las categorías migratorias (Schwenken, $2008)^{5}$.

Por todo ello, el principal objetivo de este artículo es proponer cómo podríamos reconstruir los diversos circuitos migratorios y comprender de forma más certera las diferentes formas y recorridos de los cuidados en la reproducción de personas, familias y comunidades, tanto cuando se trata de población migrante como de no migrante. Desgraciadamente, en el marco de este artículo resulta imposible hacer un profundo análisis de las diversas maneras en las que se vinculan y se configuran los circuitos y

5 Schwenken clasifica el conocimiento en tres grupos: el conocimiento tácito e irreflexivo basado en las experiencias del día a día; el saber y los significados generados por las instituciones, el mundo académico o la justicia; y el conocimiento popularizado de los medios de comunicación, guías y movimientos sociales. Esta clasificación se basa en la conceptualización del conocimiento de género de Dölling y Yresen (2005), que lo conciben como el conjunto de negociaciones sociales explícitas e implícitas sobre los significados y las relaciones de género. 
las modalidades migratorias; así pues, me limitaré a señalar algunas maneras en las que podría realizarse. Para ello me basaré principalmente en investigaciones previas realizadas en Estados Unidos sobre minorías racializadas y la aparición de una reproducción social globalizada, así como en estudios actuales del continente asiático en torno a los circuitos migratorios generizados.

Así, en el primer apartado haré un resumen del debate preliminar sobre el trabajo doméstico remunerado y la primera fase de la incorporación de mujeres migrantes al análisis de la reproducción social en la década de los noventa. El segundo apartado del artículo estará dedicado a ver cómo las mujeres migrantes y de minorías étnicas han sido incorporadas a las teorizaciones de la reproducción social, no sólo a través de flujos de empleo sino también como resultado de otros flujos, especialmente los familiares, que a su vez contribuyen a la reproducción social de personas, hogares y comunidades. La importancia del empleo de mujeres migrantes y de minorías étnicas en la mercantilización y ampliación geográfica de la reproducción social puede verse en los trabajos estadounidenses a lo largo de la década de los noventa (Colen, 1995; Nakano Glenn, 1992). Asimismo, la confluencia de diversos flujos migratorios, como el empleo y el matrimonio, tanto a nivel regional como global, ha sido estudiada más recientemente por autoras asiáticas (Constable, 2009; Lan, 2008; Palriwala y Uberoi, 2005). A pesar de que estas perspectivas no han sido tan utilizadas en el ámbito europeo, en el tercer apartado hago mención de algunas tendencias que están empezando a establecer vínculos entre distintos tipos de migraciones, lo cual resulta primordial para poder complejizar los cuidados o la reproducción social.

\section{LA REPRODUCCIÓN SOCIAL Y LA INCORPORACIÓN DEL EMPLEO MIGRANTE Y DE MINORÍAS}

Tal y como se ha señalado anteriormente, los debates en torno a la relación entre producción y reproducción se remontan a la década de los setenta y a la controversia sobre la falta de reconocimiento del valor añadido del trabajo en el hogar y de la reproducción de trabajadoras/es (Dalla Costa, 1973; Mackintosh, 1979; Smith, 1979). Sin embargo, los debates entre feministas sociales del Norte no tenían en cuenta el trabajo doméstico remunerado que proporcionaban las personas ajenas a la familia, como por ejemplo las y los migrantes rurales (Kofman y Raghuram, 2006). Supuestamente, el trabajo doméstico remunerado representaba una forma premoderna de trabajo que en su gran mayoría había desaparecido del mundo moderno (Coser, 1973; Yall, 2003). Así pues, el análisis del personal doméstico en Europa se dejó a quienes se dedicaban a la disciplina histórica (Sarti, 2008).

En las décadas de los setenta y ochenta, la creciente visibilidad de mujeres migrantes en la economía de los países del norte de Europa hizo que cada vez más trabajos académicos se centraran en la presencia de éstas en la manufactura, los talleres de explotación, los servicios públicos que requerían mano de obra menos cualificada (Condon y Ogden, 1991; Phizacklea, 1983; Knockey de los Reyes, citadas en Cederberg 2010) y, aunque con algunas excepciones en Francia, en el trabajo doméstico (Leonetti y Lévi, 1979). En lo que a análisis sociológicos se refiere, el 
objeto de estudio lo constituyó el trabajo reproductivo doméstico, así como el componente racializado del mismo; esto fue así en Sudáfrica (Cock, 1980), en Estados Unidos (Colen, 1995; Nakano Glenn, 1992) y en Latinoamérica (Radcliffe, 1990). En el Reino Unido, Gregson y Lowe $(1994,123)$ concluyeron que "no existía una correlación entre etnicidad, migración femenina y trabajo doméstico retribuido" en el marco de la reaparición de demanda de empleo doméstico, sobre todo de niñeros/as y limpiadoras/es, por parte de las clases medias a lo largo de la década de los ochenta Por el contrario, sostenían que la demanda proveniente de los hogares de personas de clase media y licenciadas conllevaba la reestructuración del empleo de acuerdo a la clase social. Así, afirmaban que mujeres de diferentes estratos sociales estaban volviendo al ámbito doméstico: las de clase media-baja a cuidar niños y niñas; las que habían pertenecido a la clase obrera a limpiar. Sin embargo, lo que más interesaba a Gregson y Lowe no eran los cuidados, sino el hecho de que el salario del trabajo doméstico en la década anterior había ido en alza por ser "un aspecto necesario de la reproducción social” (p. 75). Así pues, cuando mujeres migrantes y de minorías étnicas empezaron a estar presentes en los debates sobre trabajo doméstico en la Europa de los años noventa, a menudo lo estaban en calidad de trabajo reproductivo y no de cuidados (Anderson 2001).

En la década de los noventa aparecieron las teorizaciones sobre el papel de las minorías racializadas, las ya asentadas y las recién llegadas, en contextos nacionales (Colen, 1995; Nakano Glenn, 1992), así como en el seno de la globalización de la reproducción social (Truong, 1996). Entre estos trabajos, el de Nakano Glenn tuvo especial repercusión. Ella definió la reproducción $(1992,1)$ como "la creación y formación de personas en tanto que seres culturales, sociales y físicos que llevan a cabo una miríada de actividades y relaciones que, a su vez, sustentan a dichas personas en el día a día e intergeneracionalmente". En el trabajo de Glenn, la perspectiva histórica se incorporó a las dimensiones -entrelazadas, cambiantes- de género y raza de la reproducción. El estudio se centró en tres grupos de mujeres racializadas: afro-americanas, mejicanas-americanas y japonesas-americanas en diferentes zonas de Estados Unidos. A lo largo del siglo XX, debido a la creciente mercantilización, se produjo un cambio para estas mujeres, que pasaron de trabajar en el hogar a hacerlo en el sector de servicios institucionales mediobajos, como por ejemplo el de auxiliares de enfermería.

Por su parte, entre 1984 y 1986, Colen (1995) realizó trabajo de campo con mujeres caribeñas emigradas a Nueva York tras los cambios legislativos en materia de inmigración de 1965 y analizó su posición económica y familiar en cuanto a la 'reproducción estratificada'. Este concepto le permitía describir las relaciones de poder

${ }^{6}$ Probablemente llegaron a esta conclusión porque no consideraron que la población irlandesa fuera migrante. Además, los lugares en los que realizaron el trabajo de campo eran Reading, en Berkshire, y Newcastle upon Tyne, que en aquel entonces contaba con poca inmigración y minorías procedentes de las excolonias. Unos años más tarde, Cox (1999) mostró el carácter racializado del empleo doméstico en Londres, donde los puestos de trabajo del sector los ocupaban un grupo bastante heterogéneo de migrantes de varios estatus procedentes de Australia, Irlanda, Filipinas y Europa. 
por las cuales algunas categorías de personas ganan mayor sustento y apoyo (material y normativo), mientras que otras se enfrentan a obstáculos y dificultades en cuanto a su reproducción y cuidado. Los cuidados, que Colen diferenciaba de otras actividades en el hogar, representan, por tanto, sólo uno de los aspectos de la reproducción social. Además, encargarse de los cuidados al margen de otras actividades del hogar hace que éstos adquieran un mayor valor en el mercado laboral que el que tienen cuando se combinan con otras tareas domésticas, como la limpieza. De hecho, algunas de las entrevistadas por Colen que habían ocupado diferentes posiciones en este nicho laboral siguieron haciéndolo incluso después de haber obtenido el permiso de residencia, ya que no habían podido encontrar un trabajo mejor cualificado.

De esta manera, concluía la autora, la reproducción física y social que conlleva procrear, criar y socializar a niñas y niños, así como constituir y mantener los hogares desde la infancia hasta la edad adulta se logra, experimenta, valora y recompensa de distinta manera según las desigualdades que, en contextos históricos y culturales particulares, condicionan el acceso a los recursos materiales y sociales (Colen, 1995, 78). Los patrones de dichas desigualdades en la reproducción generan estratificación y, a su vez, reflejan, reproducen e intensifican las desigualdades de las que son fruto. Así, el resultante sistema transnacional de desigualdades se hace patente a nivel íntimo, diario y local tanto en los países emisores como en los receptores. Asimismo, las instituciones (estados, mercados de trabajo, familias, comunidades locales, medios de comunicación) contribuyen a que se den unos acuerdos vitales y familiares y a que algunos sean representados como legítimos y, por el contrario, otros, como marginales. Realizar el trabajo reproductivo para mujeres más adineradas y empleadas determina la posibilidad y el modo que diferentes tipos de migrantes tienen de reproducir sus propias familias. La rapidez con la que las mujeres migrantes consiguen reunificar sus familias depende en gran medida de cuál sea su condición migratoria y de qué posición ocupen en el seno del mercado laboral doméstico (vivir en el lugar de trabajo es algo que las personas indocumentadas han solido hacer con el objeto de que quien las o los empleara fuera su patrocinador/a). Otro modo que facilita la entrada de familiares de una persona migrante es tener una relación cercana con un o una ciudadana.

Colen y Nakano Glenn centraron sus análisis en el estado-nación; y, tras ellas, fue Truong (1996) quien amplió el foco a la relación que se establece entre producción y reproducción por medio de las transferencias globalizadas de empleo. En este sentido, afirmó que "no existe un sistema de producción que opere desprovisto de un sistema de reproducción, por lo que no debería resultar sorprendente que la globalización de la producción vaya acompañada de su inseparable 'otro', es decir, la reproducción”. Las transferencias de trabajo reproductivo de una clase, etnia, nación o región ya estaban empezando a suscitar interés, no obstante, Truong insistió en que los estudios que se centraran en éstas debían incluir una perspectiva mundial que pudiera explicar varias dimensiones: en primer lugar, los vacíos estructurales de trabajo reproductivo, que afectaban a diferentes clases y economías; en segundo lugar, los procesos por los cuales las transferencias tenían lugar; y, en tercero, las implicaciones de todo ello para los estados, el capital, las comunidades y los y las mismas trabajadoras reproductivas. En cuanto a los vacíos estructurales de trabajo en la reproducción, tres son las principales causas que señaló la autora: el repliegue estatal a la hora de respaldar el 
cuidado de niños, niñas y personas mayores; el aumento de mujeres en el ámbito del trabajo remunerado conforme la inflexibilidad de la división sexual del trabajo doméstico se mantenía; y la expansión del sector hotelero y los servicios sexuales asociados a éste último, por un lado, y la mayor movilidad de la fuerza de trabajo femenina, por otro (Truong, 1996, 34).

A pesar de todo, todavía a mediados de los años noventa no se lograba comprender del todo cuáles eran las consecuencias de los cambios en la organización de las diferentes formas de reproducción, así como de las transferencias de trabajo de Sur a Norte. Por su parte, Wolkowitz (2002) proporcionó una visión perspicaz de la segmentación y la estratificación de los servicios. Su objeto de estudio lo constituyeron las actividades en las cuales el servicio que se provee está relacionado con el cuerpo y cuya base la forman, simultáneamente, la incorporación de empleo migrante y la globalización de la reproducción social. Según la autora, los servicios corporales se han convertido en "sustitutos esenciales de la producción de objetos, entendidos como fuente de beneficio y empleo en sociedades prósperas", mientras que los artículos de alimentación y vestimenta necesarios para la reproducción humana se han exportado a países del tercer mundo. Wolkowitz cita una amplia variedad de actividades que considera parte del trabajo corporal remunerado: la estética, la peluquería, el cuidado, la preparación física, la medicina, la enfermería y el trabajo doméstico. Los grupos de profesionales de mayor estatus suelen dedicarse a actividades relacionadas con el control, la supervisión y la gestión, más que con el cuidado y el servicio prestado a otros cuerpos. Por el contrario, las actividades de menor estatus están relacionadas con aquello que genera rechazo, repelencia o que se ve como fuente de contaminación. Estas distinciones operan con fuerza en algunos trabajos en particular, como ocurre entre enfermeros/as y auxiliares de enfermería.

Los mercados laborales están segmentados por la clase, el sexo y la "raza": las actividades de menor rango como la enfermería, la limpieza y el cuidado en instituciones públicas y hogares están racializadas y quienes las llevan a cabo son las mujeres de 'razas subordinadas'. Aun así, las fronteras entre profesiones no siempre son fijas ya que, por ejemplo, la aparición de profesionales de enfermería con un amplio conocimiento y un buen salario puede hacer que las personas cuidadoras peor remuneradas bajen en la escala ocupacional y se dediquen a aquello que es considerado sucio y simbólicamente contaminante.

\section{LA VINCULACIÓN DE LOS CIRCUITOS LABORALES Y FAMILIARES}

La mayoría de los análisis que se ocupan de la reproducción transnacional y mundial se han centrado en los flujos laborales y, en menor medida, en las consecuencias de la estratificación que las desigualdades laborales producen en las vidas de las familias migrantes. No obstante, también podemos fijar la vista en la reproducción que se da en las propias familias migrantes (cuya reproducción se ha invisibilizado y privatizado [Arat-Koc, 2006, 88]) como resultado del matrimonio y de la reunificación familiar. De este modo, podremos construir un marco teórico que nos 
permita unir las diferentes formas de trabajo reproductivo con la reproducción de individuas, individuos, familias, generaciones y comunidades. Además, podremos establecer vínculos entre los variados circuitos migratorios interseccionales (Lan, 2008).

Palriwala y Uberoi $(2005,31)$ han señalado que la migración por matrimonio suele darse en paralelo a los flujos de la migración por empleo. A pesar de que la complejidad y la diversidad reinan entre los matrimonios transfronterizos, normalmente son las mujeres las que emigran para casarse. Tal y como manifiesta Constable (2005, 15), este patrón ilustra las 'geografías generizadas de poder', en que "las mujeres pueden aprovechar las oportunidades de movilidad que su posición social les brinda, las cuales son a veces inexistentes para los hombres". A menudo se casan con un hombre de un estatus económico superior, que es lo que la autora denomina 'hipergamia espacial o mundial'. De todas maneras, ello no quiere decir que siempre se casen con hombres de mayor educación o poder adquisitivo que ellas. En ocasiones el matrimonio puede implicar hipergamia geográfica sin que el estatus socio-económico entre en juego, como es el caso de mujeres formadas que contraen matrimonio con hombres del mundo rural, cuya instrucción es menor, en Corea del sur o Japón; o el fenómeno del matrimonio entre comunidades diaspóricas, como las mujeres vietnamitas que se casan con ciudadanos estadounidenses de origen vietnamita (Thai, 2008).

Por otro lado, la formación de los circuitos está muy influenciada por las políticas de inmigración y de asentamiento. En Asia, los países más ricos como Corea del Sur, Japón, Malasia y Taiwán han atraído a un gran número de migrantes, sin embargo, no permiten o dificultan la reunificación familiar, sobre todo a los o las migrantes con menor cualificación (Seol y Skrentny, 2009). Los matrimonios transnacionales han aumentado vertiginosamente (Douglass, 2006; Hugo, 2010), pero las políticas de inmigración hacen una clara distinción entre recibir empleo (remunerado) o cónyuges (trabajo no remunerado para el marido y, a menudo, también para los y las progenitoras en el hogar). Ambas categorías proveen trabajo reproductivo y cuidados, aunque de manera diferente, y la distinción tiene grandes implicaciones en los derechos y la ciudadanía tanto de las personas migrantes como de sus hijos e hijas (Turner, 2008). De hecho, que alguien que se dedica a un empleo de baja cualificación logre asentarse u obtener la ciudadanía en un país asiático es prácticamente imposible si la migración es por empleo en lugar de por matrimonio.

Partiendo del estrecho vínculo entre migraciones por empleo y matrimonio, los estudios de migraciones asiáticas han seguido las trayectorias desde el trabajo reproductivo remunerado al no remunerado, y viceversa, de las mujeres migrantes por empleo. Lo que se ve es que, a veces, algunas empiezan a trabajar en el mundo del espectáculo, los clubes, el trabajo sexual o como cuidadoras y acaban casándose con quienes les dan trabajo. En este sentido, el matrimonio puede constituir para algunas una estrategia para abandonar el trabajo doméstico y no cualificado, como en el caso de las filipinas que han entrado en Canadá a través del Programa de Cuidadores y Cuidadoras (Live-In Caregiver Program, en inglés; McKay, 2003). En otras ocasiones, en cambio, se da el fenómeno contrario y las mujeres pasan del trabajo no remunerado al remunerado, como cuando entran por matrimonio y al mismo tiempo o posteriormente se incorporan al mercado de trabajo que, asiduamente, es el del sector 
reproductivo. Sin embargo, cambiar un tipo de cuidado por otro puede resultar complicado, tal y como sucede en Japón con las trabajadoras del mundo del espectáculo filipinas casadas con japoneses. En el caso de estas mujeres es posible que estén cuidando también a sus progenitores/as y, en algunos casos, incluso realizando trabajo de cuidado remunerado en calidad de cuidadoras extranjeras (Siampukdee, 2011; Suzuki, 2007, 373). Aceptar un trabajo socialmente respetable es una manera de superar representaciones negativas. Así, los cuidados y la reproducción social se desempeñan, a lo largo del tiempo, tanto para personas desconocidas como para familiares, de manera remunerada y no remunerada. Por otra parte, Lan (2008) identifica un tipo de estrategia relacionada con la clase en tiempos de escasez de trabajo reproductivo: mientras que en los hogares de clase media y alta se contratan trabajadoras/es del hogar migrantes para externalizar el trabajo doméstico y de cuidados, los hogares de clase trabajadora buscan esposas extranjeras que provean dichos servicios de manera gratuita (Wang, 2007).

Uno de los marcos teóricos que permite relacionar diferentes procesos y trayectorias es el de las 'familias transnacionales' (global householding). Se trata de un ente más amplio que el meramente familiar, en el cual el matrimonio o el linaje directo no constituyen las únicas maneras de formar unidades relacionales. En los últimos años han aparecido contribuciones teóricas y empíricas relacionadas con el concepto de las familias transnacionales (Douglass, 2006; Petersen, 2010; Safri y Graham, 2010). Según Douglass (2006, 424), dicha noción da cuenta de las distintas fases del ciclo vital y hace que la reproducción social pueda seguir adelante ${ }^{7}$. Estas fases las marcan hechos y acontecimientos como el matrimonio, la adopción de hijas e hijos, el envío de éstas o éstos a estudiar fuera, así como que se asienten y vivan en otros países, la demanda de ayuda doméstica, la jubilación en pareja en un país de menor renta para ahorrar o el envío de remesas. Los trabajos que parten de este marco, a diferencia de los que se centran únicamente en el ámbito doméstico familiar, han podido establecer vínculos entre las diversas actividades del hogar y ver que las políticas de género en la reproducción social operan más allá de la esfera doméstica familiar. Asimismo, han constatado que todo ello hace que se perpetúen desigualdades económicas -y de otro tipo- materialmente constituidas y culturalmente normalizadas (Petersen, 2010). Además, el concepto de familias transnacionales también añade al análisis otros aspectos de la migración como la educación. Enviar a los y las hijas a estudiar fuera del propio país, por ejemplo de Corea o Taiwán, puede conllevar que las madres también se trasladen para cuidar de ellas y ellos (Chee, 2003). Es más, la creciente migración de estudiantes de grado superior facilita los matrimonios internacionales (Hugo, 2010) y, en todos estos casos, los cuidados son un aspecto de la reproducción social.

En Europa, a diferencia de lo realizado en Asia, la articulación de las migraciones por empleo y familia, en general, o la más específica confluencia entre el empleo

\footnotetext{
${ }^{7}$ Safri y Graham (2010) se decantan por el término ‘producción’ para referirse al momento de actividad económica del ciclo de reproducción y enumeran una serie de actividades que tienen como lugar de producción el hogar. Éstas incluyen, entre otras, cuidar a niñas, niños y personas mayores, preparar la comida, hacer la compra, limpiar, coser y mantener el hogar.
} 
reproductivo y la reproducción social han sido investigadas más bien poco. Es verdad que en la teoría y en lo que respecta al diseño de las políticas, la razón de ser de cada migración es distinta y específica. La migración familiar se sitúa dentro de lo social y, de hecho, desvinculada del ámbito económico (Kofman, 2004). Por ello se tiende a no considerarla como clave a la hora de abordar el empleo o la formación de migrantes familiares. Sin embargo, tal y como muestran Escrivà y Skinner (2006) en el caso español, el asunto que más preocupa a las y los migrantes familiares tiene que ver con lo económico y con el apoyo de las familias transnacionales y reconstituidas, sea a través de trabajo remunerado o de cuidado no remunerado; es decir, con lo que al trabajo reproductivo se refiere.

A la hora de establecer vínculos entre los diversos circuitos de migración resulta necesario destacar varias e importantes diferencias entre los estados asiáticos y europeos. La primera tiene que ver con la intensificación de la movilidad intraeuropea tras la ampliación de la Unión Europea en $2004^{8}$. Este fenómeno ha favorecido movimientos a corto y largo plazo en los que las motivaciones así como las estrategias de empleo y las familiares se han entrelazado (para el caso de las migraciones polacas se pueden ver Ryan et al., 2009; White, 2010). La apertura de espacios migratorios ha posibilitado que familias enteras se hayan asentado, a diferencia de otras personas migrantes que no pudieron hacerlo previamente y entre las cuales quien se movilizaba era uno o una de las integrantes (Morokvasic, 2004). Además, las y los progenitores de migrantes europeos pueden reunirse, realizar visitas de manera regular para ofrecerles apoyo práctico y emocional, o incluso preparar un viaje con poca antelación en casos de urgencia, si por ejemplo alguien enferma. De esta manera, gracias a los derechos de movilidad y ciudadanía, en los últimos años algunas y algunos migrantes de Europa del Este han podido eludir la desmembración familiar que se ha venido imponiendo a nacionales de países terceros.

Una segunda diferencia estriba en que, a pesar de que recientemente los criterios se han vuelto más restrictivos, los estados europeos permiten que parte de las familias de nacionales de países terceros se asienten (Kraler, 2010; SOPEMI, 2000) ${ }^{9}$. La migración familiar sigue siendo la causa más importante de migración permanente (Grillo, 2008; Kraler, 2010) y los matrimonios binacionales y transnacionales con personas de origen migrante se han convertido en mucho más comunes (Beck-

${ }^{8}$ Irlanda, Suecia y el Reino Unido abrieron sus fronteras en 2004, y otros estados del sur de Europa les siguieron en 2006-2007. Las fronteras alemanas permanecieron cerradas durante el periodo de transición de siete años que culminó el 1 de mayo de 2011.

${ }^{9}$ Urge tener en cuenta las diferencias que existen en el seno de la UE en lo referente al derecho de entrada y formación familiar. Las y los cónyuges con nacionalidad europea de los y las nacionales de países terceros (extracomunitarios) que ejercen su derecho a la movilidad suelen tener más ventajas que quienes pretenden reunificarse con quienes no gozan de dicho derecho. Aún más, las políticas de solicitud de visados hacen que las y los migrantes africanos tengan mayores dificultades que los y las extracomunitarias que no requieren de visado, como en el caso de la reproducción familiar biológica en Europa que algunas y algunos nacionales latinoamericanos llevan a cabo (Bledsoe y Sow, 2008). 
Gernsheim, 2007; Bryceson y Vuorela, 2002; Charsley, 2011; Eggebø, 2010). Sin embargo, la relación entre migrantes familiares y trabajo remunerado no ha sido estudiado, aunque González-Ferrer $(2007,2011)$ ha mostrado que, tanto en la Alemania de la década de los sesenta como en la España de la última década, la reunificación familiar se ha dado poco después de la llegada de la primera persona migrante. Asimismo, se ha constatado que las mujeres se han incorporado al mercado laboral y que, en algunos casos, eran quienes habían iniciado la migración (Erdem y Mattes, 2003; González-Ferrer, 2011). Todo ello lleva a concluir que la migración con fines laborales ha podido constituir, desde el principio, una estrategia familiar. Claro que en tiempos en que las políticas migratorias se están volviendo cada vez más restrictivas puede ser peligroso hacer hincapié en la contribución económica de migrantes familiares que, en términos de políticas, son construidas/os como dependientes (Eggebø, 2010); muy a menudo también se achaca a estas personas ser una carga para el estado de bienestar y una amenaza a la modernidad de las sociedades europeas, dada su baja participación en el mercado laboral (Roggeband y Verloo, 2007). Es decir, que denotan un exceso de alteridad u otredad en la reproducción de prácticas tradicionales (Grillo, 2007).

Una tercera diferencia en la comparación entre países asiáticos y europeos yace en el hecho de que en Europa el asentamiento lo pueden realizar personas que no sean cónyuges. Así, a pesar de que las y los progenitores constituyen una pequeña parte de la familia y de que en muchos estados tienen que demostrar que dependen de sus hijas o hijos (Kraler, 2010), desempeñan un papel muy importante en la reproducción social familiar a través del cuidado (normalmente no remunerado) de las y los nietos, mientras que sus hijas o hijos, sobre todo las mujeres, van a trabajar (Escrivà y Skinner, 2006; Escrivà, 2005). Las y los hermanos también pueden encargarse de las tareas de cuidado remunerado o hacerse cargo de los y las niñas cuando salen del colegio mientras la madre trabaja (Herrera, 2008). Existen más indicios de ello en países del sur de Europa, donde al principio, al no existir políticas de reunificación familiar, las y los miembros familiares entraban como turistas (González-Ferrer, 2011). Los estudios canadienses (Creese, Dyck, y McClaren, 2011) también han puesto en solfa las ventajas de las familias extensas -que normalmente se representan como tradicionales- en la integración exitosa de sus miembros, debido a la mayor cantidad de recursos materiales y afectivos de los que gozan. Cabe resaltar que establecer vínculos entre las migraciones familiares y laborales, sea de nacionales europeas/os o de países terceros, hace que las cadenas de cuidados y de trabajo reproductivo se vuelvan más complejas de lo que lo parecen en la bibliografía que las trata. Las familias migrantes ya establecidas o de reciente llegada en los países de acogida también necesitan cuidados y quienes cuentan con más recursos pueden a su vez emplear a otros y otras trabajadoras migrantes (Kofman y Raghuram, 2010; Krøger, 2003; Wall y São José, 2004; ver también Escrivà, 2005).

Tal y como se aprecia en la bibliografía asiática, existen algunos debates en torno a las políticas públicas que se ocupan del excesivo abuso de los matrimonios por conveniencia (de Hart, 2006), pero existen pocos datos que prueben la frecuencia con la que se produce un cambio en el estatus de empleada/o a cónyuge. De todas maneras, como ocurre en Asia, la opción del matrimonio no garantiza ni un permiso de residencia permanente ni la ciudadanía, ya que éstos se empiezan a tramitar después de 
que haya transcurrido un periodo de tiempo desde la formalización de la unión matrimonial. Asimismo, cada vez es más frecuente que el o la cónyuge deba superar pruebas de integración. Por otra parte, el matrimonio con un o una ciudadana también puede facilitar la reunificación familiar. Un estudio sobre mujeres migrantes latinoamericanas en Milán mostró que las migrantes que cuentan con lazos de unión familiares con personas italianas regularizan su estatus y obtienen la ciudadanía con mayor facilidad y, por tanto, pueden llevar a familiares y reconfigurar su grupo familiar de manera más sencilla (Bonizzoni, 2011). Ello alivia las presiones de la reproducción social de sus propias familias, aunque las y los hijos no siempre quieren reunificarse con sus madres o progenitores en un nuevo país tras un periodo de ausencia y en condiciones de vida muchas veces difíciles (ver también Herrera, 2008).

Así pues, para poder investigar las diferentes formas, orientaciones y direcciones que adquieren los cuidados hace falta, en primer lugar, adoptar una perspectiva que siga la trayectoria de la persona migrante longitudinal y espacialmente, para poder así aprehender tanto la prestación como la recepción de cuidados. Dado el reciente incremento en el empleo doméstico y de cuidados, muchos de los estudios se circunscriben al ámbito doméstico y a la demanda de trabajo, así como a las relaciones que se dan entre personas empleadoras y empleadas en un momento y espacio concreto. De este modo, los problemas que acechan la reconstitución familiar de los y las trabajadoras domésticas o cuidadoras se toman como un tema aparte. Sin embargo, relacionar empleo y familia nos permite obtener un retrato más complejo de los y las sujetas cuidadoras en un contexto amplio de reproducción social propio, y también de otras familias. Para ello, valerse del marco de las familias transnacionales (entendido como el nexo entre el ciclo vital y las diversas actividades que se incluyen en éste, también el cuidado) constituye una forma concreta de vincular los diferentes circuitos que se engloban en la reproducción social de las familias y los hogares. Dicho marco permite, además, entender que las familias migrantes y no migrantes de los países de destino son parte de la narrativa en torno al cuidado y la reproducción social. No obstante, es importante evitar que el marco de las familias transnacionales se entienda como un nexo exclusivamente introspectivo; y, por tanto, conviene analizarlo en relación a otras estructuras institucionales y de bienestar externas (Kofman, 2010; Kilkey, Lutz y Palenga-Möllenbeck, 2010; Williams, 2010). De hecho, tal y como han puesto de manifiesto los recientes estudios de reproducción social globalizada, este último aspecto se está volviendo cada vez más patente en las contradictorias y complejas manifestaciones de las trayectorias de cuidado.

\section{CONCLUSIONES}

En los últimos años, la reproducción social ha vuelto a convertirse en tema de debate entre algunos y algunas investigadoras feministas. Los últimos estudios han tomado en cuenta el papel que juega la mercantilización de los cuidados en el seno del hogar, así como las consecuencias de los cambios en las políticas del estado de bienestar. También se ha estudiado la globalización de la reproducción social y la función primordial que las mujeres migrantes han desempeñado en el desigual uso de recursos económicos y sociales, proporcionando cuidados para diferentes categorías de 
personas y tipos de familias, así como para asegurar la reproducción social -también la suya- en diferentes situaciones y transnacionalmente. Aunque existen diferencias entre el cuidado y otras actividades, uno de los aspectos más característicos de la mayor parte de la bibliografía que se ha producido a partir de la perspectiva de la reproducción social (Colen, Duffy, Nakano Glenn, Truong) es que traza divisiones entre las diferentes tareas que contribuyen a dicha reproducción social, sea en el entorno doméstico (limpieza, cuidado, cocina, socialización de las y los niños, entre otros) o en otros ámbitos. El interés en estas actividades de valor material y simbólico particular ha convergido, a su vez, con el estudio de la estratificación a la que conducen, incluyendo la posibilidad y el derecho a reconstituir la familia.

Asimismo, el concepto de familias transnacionales hace uso de la noción de reproducción social y se basa en las diferentes actividades que se desempeñan a lo largo del ciclo vital para sostener y mantener las familias, algunas de las cuales son plurisituadas y plurinacionales. Dicho concepto hace hincapié en la manera en la que se acumulan y se reparten los recursos económicos y sociales, y en el modo en que los distintos tipos de migración posibilitan o dificultan que dichos recursos se integren a lo largo del tiempo y el espacio. Cuidarse a uno o una misma, a familiares y a terceras personas sin vínculo de parentesco marca las diferentes etapas del ciclo vital que las y los miembros de la familia experimentan. La migración puede llevar a reconstituir familias, a que éstas continúen estando dispersas o a que se formen nuevos núcleos familiares. Y, a su vez, la posibilidad de reproducir familias y hogares física y socialmente depende de los derechos de movilidad, inmigración, residencia y ciudadanía de que se goce. La cada vez más numerosa y rica bibliografía en torno a las migraciones mundiales generizadas y a la reproducción social ha establecido, de forma provocadora, vínculos y trayectorias entre los aspectos reproductivos de las migraciones laborales y familiares. Ojalá también haya contribuido a cuestionar los paradigmas epistemológicos en los cuales se concebía cada uno de estos circuitos supuestamente diferentes.

Es, por tanto, el momento idóneo para que los estudios sobre cadenas y recorridos de los cuidados en Europa, tal y como lo hacen los artículos de este volumen, recalquen la complejidad y diversidad de los acuerdos en torno al cuidado y empiecen a dialogar con la abundante y reveladora bibliografía en torno a la reproducción social $^{10}$. La reproducción siempre conlleva una compleja interacción de varios actores y actrices en ámbitos distintos para que "sea garantizada a través de una variada constelación de fuentes que se incluyen en las más amplias categorías del estado, la familia, el capital y la sociedad civil” (Katz, 2001, 131). No obstante, las investigaciones sobre las cadenas mundiales de cuidado han tendido a dicotomizar la prestación y recepción de cuidados. Por un lado, la transferencia de trabajo al Norte global hace que tanto familias como otras instituciones se beneficien de un aumento en la adquisición de cuidado. Por otro, las familias del Sur tienen que solucionar los

${ }^{10}$ Se refiere al volumen 19 de la revista Social Politics de la primavera del 2012, cuyo número especial llevó el título de Transnational Care in Europe -Changing Formations of Citizenship, Family and Generation en el cual se publicó el presente artículo. Nota de la traductora. 
problemas que presenta el déficit de cuidado y la redistribución de la prestación del mismo entre las y los miembros de la familia y otros y otras cuidadoras ajenas a la familia. Precisamente, el hecho de que, independientemente del lugar el trabajo reproductivo, se asigne a ciertos grupos de personas es una cuestión a tratar en el marco de los procesos de reestructuración mundial que están ocurriendo hoy en día (Bakker y Silvey, 2008).

Tal y como se ha destacado en este artículo, proveer y recibir cuidado va más allá de la migración laboral y requiere que aprehendamos la vinculación de los diferentes circuitos de migración que, a su vez, generan configuraciones distintas y recorridos y trayectorias dinámicas. Tampoco deberíamos olvidar que las tareas de cuidado, que constituyen un aspecto de la reproducción social de las familias migrantes y no migrantes, las llevan a cabo en los diferentes lugares y ámbitos (familias, comunidades, estado, ONG) tanto personas trabajadoras poco cualificadas como cualificadas -aunque normalmente éstas últimas sean sometidas a una ardua desprofesionalización. El trabajo de cuidado está estratificado por la clase, la inmigración y el estatus de ciudadanía que, a su vez, condiciona el acceso a recursos estatales para la reproducción familiar. En este artículo no he podido tratar más de cerca estas complejísimas cuestiones y la manera en la que han contribuido a la reproducción social en diferentes estados de bienestar europeos; aunque el hecho es que los cuidados que proveen las y los migrantes hacen que esos estados de bienestar continúen funcionando, sea en el seno de las familias, en los hogares particulares o en las instituciones estatales.

\section{REFERENCIAS BIBLIOGRÁFICAS}

ADKINS, LISA \& BEVERLY, SKEGGS (2005): Feminism after Bourdieu. Oxford. Blackwell.

ANDALL, JACQUI (2003): Gender and Ethnicity in Contemporary Europe. Oxford: Berg.

ANDERSON, BRIDGET (2001): Reproductive labour and migration. 6th International Metropolis Conference, Rotterdam, Oxford University. WPTC-02-01

ARAT-KOÇ, SEDEF (2006): Whose Social Reproduction? Transnational Motherhood and Challenges to Feminist Political Economy. En Luxton M. \& Bezanson K. (eds.), Social Reproduction: Feminist Political Economy Challenges Neo-Liberalism. Montreal: McGill-Queen's University Press.

BAKKER, ISABELLA (2007): Social Reproduction and the Constitution of a Gendered Political Economy. New Political Economy, 12, 4, 541-56.

BAKKER, ISABELLA \& GILL, STEPHEN (eds.) (2003): Power, Production and Social Reproduction. London and New York: Macmillan-Palgrave.

BAKKER, ISABELLA \& SILVEY, RACHEL (eds.) (2008): Beyond States and Markets: the Challenges of Social Reproduction. Abingdon and New York: Routledge. 
BECK-GERSHEIM, ELISABETH (2007): Transnational Lives, Transnational Marriages: A Review of Evidence from Migrant Communities in Europe. Global Networks, 7(3), 271-288.

BEDFORD, KATE \& RAI, SHIRIN (2010): Feminists Theorize Political Economy. Journal of Women in Culture and Society, 36(1), 1-18.

BENERIA. LOURDES (1979): Reproduction, Reproduction and the Sexual Division of Labor. Cambridge Journal of Economics, 3, 203-225.

BENERIA, LOURDES (1981): Reproducción, producción y división sexual del trabajo. Mientras Tanto, 6, 47-84.

BENERIA, LOURDES (2006): Trabajo productivo/reproductivo, pobreza, y la globalización de la reproducción. Consideraciones teóricas. Mientras Tanto, 100, 89-107.

BENERIA, LOURDES (2007): Paid/unpaid work and the Globalization of Reproduction. GEM-IWG Working Paper 07-01.

BERGERON, SUZANNE (2011): Economics, performativity, and social reproduction in global development. Globalizations, 8(2), 151-161.

BJEREN, GUNILLA (1997): Gender and Reproduction. En Hammar, T., Brochmann, G., Tamas, T. \& Faist, T. (eds.), International Migration, Immobility and Development, 219-246. Oxford: Berg.

BLEDSOE, CAROLINE \& SOW, PAPA (2008): Family Reunification Ideals and the Practice of Transnational Reproductive Life among Africans in Europe. Rostock, Germnay: Max Planck Institute for Demographic Research

BONIZZONI, PAOLA (2011): Civic Stratification, Stratified Reproduction and Family Solidarity Strategies of Latino families in Milan. En Kraler, A., Kofman, E., Kohli, M. \& Schmoll, C. (eds.), Gender, Generations and the Family in International Migration, eds. Amsterdam: University of Amsterdam Press.

BRYCESON, DEBORAH \& VUORELA, ULLA (2002): The Transnational Family: new European frontiers and global networks. Oxford: Berg.

CEDERBERG, MAJA (2010): Gender (In)equality and Ethnic Boundaries. Gender, Migration and Ethnicity in the Swedish Labour Market and Society. En Slany, K., Kontos, M. \& Liapi, M. (eds.), Women in New Migrations. Current Debates in European Societies, 121-39. Cracow: Jagiellonian University Press.

CHARSLEY, KATHERINE (ed.) (2011): Transnationalism, Migration and Marriage. London: Routledge

CHEE, MARIA (2003): Migrating for the Children: Taiwanese American women in Transnational Families. En Piper, N. \& Roces, M. (eds.), Wife or Worker? Asian Women and Migration, 137-56. Lanham Maryland: Rowman and Littlefield.

COCK, JACKLYN (1980): Maids and Madams: A Study in the Politics of Exploitation. Johannesburg: Ravan Press. 
COLEN, SHELEE (1995): Like a Mother to Them: Stratified Reproduction and West Indian Childcare Workers and Employers in New York. En Ginsburg, F. \& Rapp, R. (eds.), Conceiving the New World Order: The Global Politics of Reproduction, 78102. Berkeley: University of California Press.

CONDON, STEPHANIE \& OGDEN, PHILIP (1991): Emigration from the French Caribbean: The Origins of an Organized Migration. International Journal of Urban and Regional Research, 15(4), 505-23.

CONSTABLE, NICOLE (2005): Introduction: Cross-border Marriages, Gendered Mobility and Global Hypergamy. En Constable, N. (ed.), Cross-Border Marriages: Gender and Mobility in Transnational Asia, 1-16. Philadelphia: University of Pennsylvania Press.

CONSTABLE, NICOLE (2009): The Commodification of Intimacy: Marriage, Sex, and Reproductive Labor. Annual Review of Anthropology, 38, 49-64.

COSER, LEWIS (1973): Servants: The Obsolescence of an Occupational Role. Social Forces, 52(1), 31-40.

COX, ROSIE (1999): The Role of Ethnicity in Shaping the Domestic Employment Sector in Britain. En Momsen, J. (ed.), Gender, Migration and Domestic Service, 131-44. London: Routledge.

CREESE, GILLIAN; DYCK, ISABEL \& MCCLAREN, TIGER (2011): The Problem of 'Human Capital': Gender, Place and Immigrant Household Strategies of Reskilling in Vancouver, Canada. En Kraler, A., Kofman, E., Kohli, M. \& Schmoll, C. (eds.), Gender, Generations and the Family in International Migration, 139-160. Amsterdam University of Amsterdam Press.

DALla COSTA, M. (1973): The Power of Women and the Subversion of the Community. Bristol: Falling Wall Press.

DALY, MARY. ED. (2001): Care Work. The Quest for Security. Geneva: International Labour Office.

DE HART, BETTY (2006): Introduction: The marriage of convenience in European immigration law. European Journal of Migration and Law, 8(3-4), 251-62.

DOUGLASS, MIKE (2006): Global Householding in Pacific Asia. International Development Planning Review, 28(4), 421-445.

EGGEBØ (2010): The Problem of Dependency: Immigration, Gender and the Welfare State. Social Politics, 17(3), 295-322.

ELSON, DIANE (2000): Gender at the Macroeconomic Level. En Cook, J., Roberts, J. \& Waylen, G. (eds), Towards a Gendered Political Economy. Basingstoke: Macmillan Press.

ENGLAND, PAULA \& FOLBRE, NANCY (2003): Contracting for Care. En Ferber, M. \& Nelson, J., Feminist Economics Today: Beyond Economic Man. Chicago: University of Chicago Press. 
ERDEM, ESRA \& MATTES, MONIKA (2003): Gendered Policies - Gendered Patterns: Female Labour Migration from Turkey to Germany from the 1960s to the 1990s. En Phliger, R., Schonwalder, K. \& Triadafilopoulos, T. (eds), European Encounters. Migrants, migration and European societies since 1945. Aldershot: Ashgate

ESCRIVÀ, ANGELES (2005): Aged Global Care Chains: A Southern-European Contribution to the Field. Comunicación presentada en el International Conference on Migration and Domestic Work in Global Perspective. Wassenaar (The Netherlands), 26-29 May.

ESCRIVÀ, ANGELES \& SKINNER, EMMELINE (2006): To Spain at an Advanced Age. Generation Review, 16(2), 8-15.

GONZÁLEZ-FERRER, AMPARO (2007): The Process of Family Reunification among Original Guest-workers in Germany. Zeitschrift für Familienforschung, 19(1). 10-33.

GONZÁLEZ-FERRER, AMPARO (2011): Family Dimensions of Immigration to Spain. En Kraler et al. (eds.), Gender, Generations and the Family in International Migration, 191-217. Amsterdam: University of Amsterdam Press.

GREGSON, NICKY \& LOWE, MICHELLE (1994): Servicing the Middle Classes: Class, gender and waged domestic labour in contemporary Britain. London: Routledge.

GRILLO, RALPH (2007): An Excess of Alterity? Debating Difference in a Multicultural Society. Ethnic and Racial Studies, 30(6), 979-998.

GRILLO, RALPH (ed.) (2008): The Family in Question. Amsterdam: University of Amsterdam Press.

HERRERA, GIOCONDA (2008): States, work and social reproduction through the lens of migrant experience: Ecuadorian domestic workers in Madrid. En Bakker, I. \& Silvey, R. (eds.), Beyond States and Markets: The Challenges of Social Reproduction, 93-107. Abingdon and New York: Routledge.

HILL MAHER, KRISTIN (2004): Globalized Social Reproduction: Women Migrants and the Citizenship Gap. En Brysk, A. \& Shafir, G. (eds.), People Out of Place. Globalization, Human Rights and the Citizenship Gap, 131-52. London: Routledge.

HOCHSCHILD, ARLIE R. (2001): Global Care Chains and Emotional Surplus Value. En Giddens, A. \& Hutton, W. (eds.), In On the Edge: Living with Global Capitalism, 130-46. London: Jonathan Cape.

HUGO, GRAHAM (2010): The Future of Migration Policies in Asia Pacific. En IOM Background Paper World Migration Report 2010 http://publications.iom.int/bookstore/free/WMR2010_migration_policies_asiapacific.pdf

KATZ, CINDY (2001): Vagabond Capitalism and the Necessity of Social Reproduction. Antipode, 33(4), 709-28. 
KILKEY, MAJELLA; LUTZ, HELMA \& PALENGA-MÖLLENBECK, EWA (2010): Introduction: Domestic and Care Work at the Intersection of Welfare, Gender and Migration Regimes: European Experiences. Social Policy and Society, 9(3),379-84.

KOFMAN, ELEONORE (2004): Family-related Migration: A Critical Review of European Studies. Journal of Ethnic and Migration Studies, 30(2), 243-62.

KOFMAN, ELEONORE (2010): Gendered Migrations and the Globalisation of Social Reproduction and Care: New Dialogues and Directions. En Schrover, M. \& Yeo, E. (eds.), Gender, Migration and the Public Sphere, 118-39. Abingdon and New York: Routledge.

KOFMAN, ELEONORE \& RAGHURAM, PARVATI (2006): Gender and global labour migrations: incorporating skilled workers. Antipode, 38(2), 282-303.

KOFMAN, ELEONORE \& RAGHURAM, PARVATI (2010): The implications of migration for gender and care regimes in the South. En Hugo, K. \& Piper, N. (eds.), South-South migration: Implications for Social Policy and Development, 4683. Palgrave.

KRØGER, TEPPO (ed.) (2003): New Kinds of Families, New Kinds of Social Care. SOCCARE Project Families, Work and Social Care in Europe. A Qualitative Study of Care Arrangements in Finland, France, Italy, Portugal and the UK. Final report.

KRALER, ALBERT (2010): Civic Stratification, Gender and Family Migration Policies in Europe. Final Report. Vienna: BMWF/ICMPD. Available at: http://research.icmpd.org/1445.html

LAN, PEI-CHAN (2008): New Global Politics of Reproductive Labor: Gendered Labor and Marriage Migration. Sociology Compass, 26, 1801-15.

LASLETT, BARBARA \& BRENNER, JOHANNA (1989): Gender and Social Reproduction. Historical Perspectives. Annual Review of Sociology, 15, 381-404.

LEON, MARGARITA (2010): Migration and Care Work in Spain: The Domestic Sector Revisited. Social Policy and Society, 9(3), 409-18.

LEONETTI, ISABELLE \& LÉVI, FLORENCE (1979): Femmes et Immigrées. Paris: La Documentation Française.

LISTER, RUTH (2006): Children (but not Women) First: New Labour, Child Welfare and Gender. Critical Social Policy, 26 (2), 315-35.

LUXTON, MEG \& BEZANSON, KATE (2006): Social reproduction: feminist political economy challenges neo-liberalism. Montreal: McGill-Queen's Press.

MACKINTOSH, MAUREEN (1979): Domestic labour and the household. En Burman, S. (ed.), Fit Work for Women. London: Croom and Helm.

MCKAY, DEIDRE (2003): Filipinas in Canada - Deskilling as a Push towards Marriage. En Piper, P. \& Roces, M., Wife or Worker? Asian Women and Migration, 23-51. Lanham Maryland: Rowman and Littlefield. 
MANALANSAN, MARTIN (2006): Queer Intersections: Sexuality and Gender in Migration Studies. International Migration Studies, 40(1), 224-49.

MISRA, JOYA, WOODRING, JONATHAN \& MERZ, SABINE (2006): The Globalization of Carework: Immigration, Economic Restructuring, and the WorldSystem. Globalization, 3(3), 317-332.

MOLYNEUX, MAXINE (1979): Beyond the Domestic Labour Debate. New Left Review, 116, 1-15.

MOMSEN, JANET (ed.) (1999): Gender, Migration and Domestic Service. London: Routledge.

MOROKVASIC, MIRJANA (2004): Settled in Mobility: Engendering Post-wall Migration in Europe. Feminist Review, 77, 7-25.

NAKANO GLENN, EVELYN (1992): From Servitude to Service Work. Historical Continuities in the Racial Division of Paid Reproductive Work Signs. Journal of Women in Culture and Society, 18(1), 1-43.

PALRIWALA, RAJNI \& UBEROI, PATRICIA (2005): Marriage and Migration in Asia: Gender Issues. Indian Journal of Gender Studies, 12, 5-29

PARRENAS, RHACEL (2001): Servants of Globalization: Women, Migration and Domestic Work. Stanford University Press.

PEREZ OROZCO, AMAIA (2010): Global Perspectives on the Social Organization of Care in Times of Crisis: Assessing the Situation. Gender, Migration and Development Working Paper 5, INSTRAW UN.

PETERSEN, SPIKE V. (2003): A Critical Rewriting of Global Political Economy: Integrating Reproductive, Productive and Virtual Economies. London and New York: Routledge

PETERSEN, V. SPIKE (2010): Global Householding amid Global Crises. Politics and Gender, 6(2), 271-281.

PHIZACKLEA, ANNIE (ed.) (1983): One Way Ticket; Migration and Female Labour. London: Routledge.

RADCLIFFE, SARAH (1990): Ethnicity, patriarchy and incorporation into the nation: female migrants as domestic servants in Peru. Environment and Planning. Society and Space, 8, 379-93.

REAY, DIANE (2004): Gendering Bourdieu's Concept of Capitals? Emotional Capital, Women and Social Class. En Adkins, L. \&. Skeggs. B. (eds.), Feminism After Bourdieu, 57-74. Oxford: Blackwell.

ROGGEBAND, CONNY \& VERLOO, MIEKE (2007): Dutch Women are Liberated, Migrant Women are a Problem: The Evolution of Policy Frames on Gender and Migration in the Netherlands, 1995-2005. Social Policy and Administration, 41(3), 271-88. 
SAFRI, MALIKA \& GRAHAM, JULIE (2010): The Global Household: Toward a Feminist Postcapitalist International Political Economy. Signs. Journal of Women in Culture and Society, 36(1), 99-125.

SALES, ROSEMARY, RYAN, LOUISE, TILKI, MARY \& SIARA, BERNADETTE (2009): Family Strategies and Transnational Migration: Recent Polish Migrants in London. Journal of Ethnic and Migration Studies, 35 (1), 61-77.

SARTI, RAFFAELLA (2008): The Globalization of Domestic Service -an Historical Perspective. En Helma, L. (ed.), Migration and domestic labour: A European perspective on a global theme, 77-98. Farnham: Ashgate.

SCHWENKEN, HELEN (2008): Beautiful Victims and Sacrificing Heroines: Exploring the Role of Gender Knowledge in Migration Policies. Signs. Journal of Women in Culture and Society, 33(4), 770-76.

SEOL, DONG-HOON \& SKRENTNY, JOHN (2009): Why is There so Little Migrant Settlement in East Asia? International Migration Review, 43(3), 578-620.

SIAMPUKDEE, USAMARD (2011): The Politics of Importing Health and Social Care Workers: Japan's Economic Partnership Agreements with Southeast Asian Countries. PhD University of Leeds.

SMITH, PAUL (1979): Domestic labour and Marx's theory of value. En Kuhn, A. \& Wolpe, A. (eds.). Feminism and Materialism. London: Routledge and Kegan Paul.

SOPEMI (2000): Comparative Analysis of the Legislation and the Procedures Governing the Immigration of Family Members in Certain OECD Countries. En Trends in International Migration, 105-26, Paris: OECD.

SUZUKI, NOBUE (2007): Carework and Migration: Japanese Perspectives on the Japan-Philippines Economic Partnership Agreement. Asian and Pacific Migration Journal, 16(3), 357-381.

THAI, HUNG CAM (2008): For Better or For Worse. Vietnamese International Marriages in the New Global Economy. Rutgers University Press.

TRUONG, THANH-DAM (1996): Gender, International Migration and Social Reproduction: Implications for Theory, Policy, Research and Networking. Asian and Pacific Migration Journal, 5(1), 27-51.

TURNER, BRYAN (2008): 'Citizenship, Reproduction and the State: International Marriage and Human Rights. Citizenship Studies, 12(1), 45 - 54.

UNRISD (2009): The political and social economy of care. Report of the UNRISD Conference 6 March 2009, New York: Barnard College, Columbia University.

VENKEN, MACHTELD, BEYERS, LEEN \& GODDEERIS, IDESBALD (2009): Families, Foreignness, Migration: Now and Then. The History of the Family, 14(2), 125-31.

VOGEL, LISE (1995): Woman Questions: Essays for a Materialist Feminism. New York: Routledge. 
WALL, KARIN \& SÃO JOSÉ, JOSÉ (2003): Immigrant Families, Work and Social Care. A Qualitative Comparison of Care Arrangements in Finland, Italy, the U.K., France and Portugal. Soccare WP4.

WALL, KARIN \& SÃO JOSÉ, JOSÉ (2004): Managing work and care: A difficult challenge for immigrant families. Social Policy and Administration, 38(6), 591-621.

WALTON-ROBERTS, MARGARET (en prensa). Contextualizing the Global Nursing Chain: International Migration and the Status of Nursing in Kerala, India. Global Networks,

WANG, HONG-ZEN (2007): Hidden Spaces of Resistance of the Subordinated: Case Studies from Vietnamese Female Migrant Partners in Taiwan. International Migration Review, 41, 706-27.

WHITE, ANNE (2010): Polish Families and Migration Since EU Accession. Bristol: Policy Press.

WILLIAMS, FIONA (2010): Migration and Care: Themes, Concepts and Challenges. Social Policy and Society, 9(3), 385-96.

WOLKOWITZ, CAROL (2002): The Social Relations of Body Work. Work, Employment and Society, 16 (3), 497-510.

YEATES, NICOLA (2004): Global Care Chains. Critical Reflections and Lines of Enquiry. International Feminist Journal of Politics, 6(3), 369-91.

YEATES, NICOLA (2009): Globalizing Care Economies and Migrant Workers: Explorations in Global Care Chains. UK: Palgrave MacMillan. 